\title{
Comportamento alimentar em adolescentes de Juiz de Fora
}

\author{
Eating habits in adolescents of Juiz de Fora \\ El comportamiento alimentario en los adolescentes de Juiz de Fora
}

\author{
Leonardo de Sousa Fortes* \\ Maria Elisa Caputo Ferreira* \\ Ana Carolina Soares Amaral ${ }^{* *}$
}

\begin{abstract}
Resumo
O objetivo deste estudo foi avaliar o comportamento alimentar de adolescentes de Juiz de Fora-MG, comparando-o de acordo com o sexo e o estado nutricional. Participaram 322 adolescentes com idades entre 10 e 19 anos, de ambos os sexos. Utilizou-se o eating attitudes test (EAT-26) para avaliar o comportamento alimentar. Aferiram-se o peso e a estatura para calcular o índice de massa corporal (IMC). O estado nutricional foi obtido pelos pontos de corte do IMC. Conduziu-se análise Manova para comparar os três fatores do EAT-26 em razão do sexo e estado nutricional. Os resultados demonstraram diferenças estatisticamente significativas nas subescalas dieta e autocontrole oral $(\mathrm{p}<0,05)$ conforme o sexo. Ademais, encontraram-se diferenças significativas nas subescalas dieta e bulimia e preocupação com alimentos $(\mathrm{p}<0,05)$ segundo o estado nutricional. Concluiu-se que o comportamento alimentar foi diferente entre os sexos e grupos de estado nutricional.
\end{abstract}

Palavras-chave: Comportamento alimentar. Transtornos alimentares. Adolescentes.

\begin{abstract}
The aim of this study was to evaluate the eating habits of adolescents in Juiz de Fora-MG, comparing them according to sex and nutritional state. Participants 322 adolescents aged between 10 and 19 years, of both sexes. We used the Eating Attitudes Test (EAT-26) to evaluate the eating habits. Measure body weight and height to calculate the body mass index (BMI). Nutritional status was obtained by points BMI cutoff. Manova analysis
\end{abstract}

\footnotetext{
Texto recebido em março 2012 e aprovado para publicação em julho de 2014.

- Doutor em Psicologia pela Universidade Federal de Juiz de Fora (UFJF), membro do Núcleo de Educação Física e Ciências do Esporte do Centro Acadêmico de Vitória - Universidade Federal de Pernambuco (UFPE). Endereço: Rua Clóvis Beviláqua, 163, ap. 1003 - Madalena, Recife-PE, Brasil. CEP: 50710-330.E-mail: leodesousafortes@hotmail.com.

* Doutora em Psicologia pela UFJF, membro do Instituto Federal de Ciência e Tecnologia do Sudeste de Minas Gerais. Endereço: Av. Luz Interior, no 360 - Bairro Estrela Sul, Juiz de Fora-MG, Brasil. CEP: 36030-776. E-mail: acsamaral@hotmail.com.

*** Doutora em Educação pela Universidade de São Paulo, membro do Departamento de Fundamentos da Educação Física da Universidade Federal de Juiz de Fora (UFJF). Endereço: Rua José Lourenço Kelmer, s/n. ${ }^{\circ}$ - Martelos, Juiz de Fora-MG, Brasil. CEP: 36036-330. E-mail: caputoferreira@terra.com.br.
} 
was conducted to compare the three factors of the EAT-26 according to sex and nutritional status. The results showed differences statistically significant on the scales Self-control oral and Diet $(p<0.05)$ between the sexes. Furthermore, we found significant differences in the subscales Diet and Bulimia and concern with food $(\mathrm{p}<0.05)$ according to nutritional status. It was concluded that eating habits were different between sexes and nutritional status groups.

Keywords: Eating behavior. Eating disorders. Adolescents.

\section{Resumen}

El objetivo de este estudio fue evaluar el comportamiento alimentario de los adolescentes en Juiz de Fora-MG, comparándolos en función del sexo y el estado nutricional. Participaron 322 adolescentes de entre 10 y 19 años, de ambos sexos. Se utilizó el Eating Attitudes Test (EAT-26) para evaluar el comportamiento alimentario. Se registraron la altura y el peso para calcular el índice de masa corporal (IMC). El estado nutricional se obtuvo por los puntos de corte del IMC. Se realizó el análisis Manova para comparar los tres factores EAT-26 según el sexo y el estado nutricional. Los resultados mostraron diferencias estadísticamente significativas en los apartados de dieta y autocontrol oral $(\mathrm{p}<0,05)$ según el sexo. Además, se encontraron diferencias significativas en los apartados de dieta y bulimia y preocupación con los alimentos $(\mathrm{p}<0,05)$ según el estado nutricional. Se concluyó que el comportamiento alimentario fue diferente entre los sexos y los grupos del estado nutricional.

Palabras clave: El comportamiento alimentario. Trastornos de la alimentación. Adolescentes.

\section{Introdução}

Cegundo a Organização Mundial de Saúde (WHO, 2007), adolescência é um período compreendido entre os 10 e 19 anos de idade. Nessa fase, o jovem vivencia mudanças psicológicas e sociais, além de passar por alteraçóes morfológicas decorrentes da puberdade (Martins, Pelegrini, Matheus \& Petroski, 2010; Scherer, Martins, Pelegrini, Matheus \& Petroski, 2010). Durante essa etapa, meninas geralmente apresentam aumento do perfil lipídico, ao contrário do sexo masculino, que costuma demonstrar aumento da massa livre de gordura (Siervogel, Dmerath, Schubert, Remsberg \& Chumlea, 2003).

Essas alterações podem acentuar a preocupação que o adolescente tem com seu corpo. Estudos têm apresentado elevada prevalência de insatisfação corporal em jovens escolares brasileiros (Castro, Levy, Cardoso, Passos \& Sardinha, 2010; 
Duca, Garcia, Sousa, Oliveira \& Nahas, 2010; Scherer et al., 2010). Sabe-se que a depreciação com o peso e com a aparência física pode predispor os jovens a desenvolverem hábitos deletérios à saúde na tentativa de modificarem suas morfologias corporais (Fortes, Morgado \& Ferreira, 2013; Miranda, Conti, Bastos \& Ferreira, 2011; Oliveira \& Hutz, 2010), gerando comportamentos alimentares inadequados e outras práticas prejudiciais à saúde (Alvarenga, Scagliusi \& Phillippi, 2011; Ximenez, Colares, Bertulino, Couto \& Sougey, 2011). Autores ressaltam que a autoindução de vômitos, o uso de medicamentos (diurético e laxantes) para emagrecimento e a restrição alimentar são sintomas comuns de síndromes psicológicas como a anorexia nervosa (AN) e a bulimia nervosa (BN) (Alvarenga et al., 2011; Bernardi, Harb, Levandovski \& Hidalgo, 2009; Ekeroth \& Birgegård, 2014; Rossi, Moreira \& Rauen, 2008).

Estudos têm demonstrado que o sexo feminino é mais acometido por influências negativas advindas da cultura e, como consequência, apresentam maiores riscos para os transtornos alimentares (Alvarenga et al., 2011; Castro et al., 2010; Fortes, Almeida, Cipriani \& Ferreira, 2014; Miranda et al., 2011; Rossi et al., 2008; Scherer et al., 2010). Estudos recentes têm comprovado que jovens do sexo feminino apresentam maior chance de desenvolverem comportamentos alimentares inadequados quando comparadas aos do sexo masculino (Fortes, Kakeshita, Gomes, Almeida \& Ferreira, 2014; Johnson, Crosby, Engel, Mitchell \& Powers, 2004; Santos, Tassitano, Nascimento, Petribu \& Cabral, 2011).

Ademais, parece que o estado nutricional também pode influenciar negativamente os hábitos alimentares entre os adolescentes. Autores têm identificado que indivíduos com sobrepeso/obesidade apresentam maior frequência de alimentação desordenada quando comparados a sujeitos eutróficos (Scherer et al., 2010; Martins et al., 2010). Entretanto não foram encontrados na literatura estudos que tenham confrontado a variável comportamento alimentar em razão das classificações de estado nutricional, nem entre jovens do sexo feminino e masculino. Diante do exposto, o objetivo deste estudo foi avaliar o comportamento alimentar de adolescentes de Juiz de Fora-MG, comparando-o conforme o sexo e o estado nutricional.

\section{Metodo}

População e amostra

Trata-se de pesquisa transversal realizada em duas escolas, uma privada e outra pública, com adolescentes de ambos os sexos, com idade entre 10 e 19 anos, residentes na cidade de Juiz de Fora-MG. Segundo informações do Censo Escolar 
de 2010 (Minas Gerais, 2011), esse Município apresentava cerca de 80 mil jovens nessa faixa-etária, matriculados em escolas privadas e públicas. Para o cálculo amostral, adotou-se prevalência de comportamento alimentar inadequado de 30\% (Scherer et al., 2010; Martins et al., 2010), 5\% de erro amostral e 95\% de confiança, chegando-se ao tamanho amostral de 322 adolescentes. Participaram do estudo 395 jovens, sendo que 33 destes foram excluídos por não responderem aos questionários em sua totalidade e, ou, não participaram das aferições antropométricas, obtendo-se então uma amostra de 362 participantes. Para inclusão no estudo, o adolescente necessitava estar regularmente matriculado nas instituições de ensino fundamental/médio no Município de Juiz de ForaMG, ter disponibilidade para responder a questionários e realizar medidas antropométricas, além de apresentar o termo de consentimento livre e esclarecido (TCLE) assinado pelo responsável.

O projeto foi aprovado pelo Comitê de Ética e Pesquisa em Seres Humanos da Universidade Federal de Juiz de Fora, recebendo protocolo 2282.022.2011, com parecer número 022/2011, de acordo com a resolução 196/1996 do Conselho Nacional de Saúde.

\section{Instrumentos}

Para avaliar o comportamento alimentar, utilizou-se o Eating Attitudes Test em sua versão abreviada (EAT-26) (Garner, Olmsted, Bohr \& Garfinkel, 1982). Esse é um instrumento de autopreenchimento, com 26 questôes em escala do tipo likert, variando de 0 (nunca) a 3 (sempre) pontos, sendo que o item 25 apresenta escore invertido $(0=$ sempre, muitas vezes e às vezes; $1=$ poucas vezes; $2=$ quase nunca; 3 = nunca). Essas questôes buscaram avaliar dieta, sintomas de bulimia, restrição alimentar e influência do ambiente sobre os hábitos alimentares, e foram distribuídas em três fatores:

a) dieta - dizia respeito à recusa patológica a alimentos com alto teor calórico e preocupação com aparência física;

b) bulimia e preocupação com os alimentos - referia-se a episódios de compulsão alimentar, seguido por comportamentos purgativos para perda/controle de peso corporal e;

c) autocontrole oral - refletiu o autocontrole em relação à comida e avaliou forças ambientais e sociais estimulantes à ingestão alimentar. 
Escore maior ou igual a 20 representa indivíduos com riscos para transtorno alimentar (Garner et al., 1982). O EAT-26 foi validado no Brasil para adolescentes do sexo feminino (Bighetti, Santos, Santos \& Ribeiro, 2004) e masculino (Fortes, Amaral, Conti, Cordás \& Ferreira, 2016), apresentando alpha de Cronbach de 0,82 e 0,88, respectivamente. Para esta amostra, calculouse a consistência interna, identificando-se valores de 0,89 e 0,86 para meninas e meninos, respectivamente.

\section{Avaliação antropométrica}

A massa corporal foi mensurada por uma balança digital portátil da marca Tanita, com precisão de $100 \mathrm{~g}$ e capacidade máxima de $200 \mathrm{~kg}$. Utilizou-se de estadiômetro portátil, com precisão de $0,1 \mathrm{~cm}$ e altura máxima de $2,20 \mathrm{~m}$, da marca Welmy, para mensurar a estatura dos adolescentes.

O índice de massa corporal [IMC = massa corporal $(\mathrm{kg}) /$ estatura $\left.\left(\mathrm{m}^{2}\right)\right]$ foi usado como indicador de estado nutricional. A classificação do IMC obedeceu aos critérios da Organização Mundial de Saúde (World Health Organization [WOH], 2007), que propõe a classificação em baixo peso, peso normal, sobrepeso e obeso, de acordo com os percentis (5, 85 e 95), em relação à idade cronológica.

\section{Procedimentos}

Inicialmente, a direção de cada escola foi contatada pelos pesquisadores, que explicaram os objetivos e procedimentos do projeto. Após aprovação inicial, distribuiu-se o TCLE aos alunos, que foram esclarecidos sobre todos os procedimentos necessários para realização da pesquisa.

Os dados foram coletados entre os meses de março e junho de 2011, com o mínimo de duas etapas. No primeiro momento, em sala de aula, após a entrega do TCLE devidamente assinado pelos responsáveis, os alunos responderam individualmente ao EAT-26 e a um cabeçalho de identificação contendo nome (opcional), sexo e idade. Os questionários foram aplicados por um mesmo pesquisador (LSF), que padronizou as orientaçôes verbais. Os sujeitos do estudo não comunicavam entre si e não houve limite de tempo para preenchimento do EAT-26.

Com a entrega do questionário, o aluno foi encaminhado para uma sala cedida pela escola para mensuração de peso e estatura. Para tanto, o jovem deveria estar trajando uniforme para a aula de Educação Física e descalço. 


\section{Análise dos dados}

Utilizaram-se de medidas de tendência central (média e desvio padrão) e frequências para descrição das variáveis do estudo. Conduziu-se análise multivariada de covariância (manova) para comparar os três fatores do EAT-26 (dieta, bulimia e preocupação com os alimentos, autocontrole oral) conforme o sexo (covariáveis IMC e idade) e estado nutricional (covariável idade). Realizouse análise univariada de covariância (ancova) para comparar o escore total do EAT-26 em razão do sexo (covariáveis IMC e idade) e estado nutricional (covariável idade). Todos os dados foram tratados no software SPSS, versão 17.0, adotando-se nível de significância de 5\%.

\section{Resultados}

Participaram da pesquisa 199 (55\%) meninas e 163 (45\%) meninos, sendo que $276(76,2 \%)$ estavam matriculados em instituição pública e $86(23,8 \%)$ eram de escola particular. A média de idade entre as adolescentes femininas foi de $12,72( \pm 1,59)$ e de $12,65( \pm 1,69)$ entre os masculinos. O IMC derivado do peso e estatura aferidos foi de $19,77( \pm 3,66)$ e $19,97( \pm 3,51)$, respectivamente.

Em relação à comparação dos três fatores do EAT-26 segundo o sexo, identificaram-se diferenças estatisticamente significativas nas subescalas dieta $(F(6,356)=39,50, p=0,001)$ e autocontrole oral $(F(6,356)=24,66, p=0,01)$, fato não ocorrido para a subescala bulimia e preocupação com os alimentos $(F(6$, $356)=3,77, p=0,23)$, conforme elucidado na tabela 1 . Além disso, encontrou-se maior pontuação no escore total do EAT-26 no sexo feminino quando comparado ao masculino $(F(2,360)=41,80, p=0,001$, tabela 1$)$.

Tabela 1. Comparação do escore total e dos três fatores do EAT-26 de adolescentes de Juiz de Fora segundo sexo

\begin{tabular}{|l|c|c|}
\hline \multicolumn{1}{|c|}{ Variável } & \multicolumn{2}{|c|}{ Sexo } \\
\hline & Feminino $(n=199)$ & Masculino $(n=163)$ \\
\hline & M (DP) & M (DP) \\
\hline Dieta & $8,44( \pm 0,45)^{\mathrm{a}}$ & $6,13( \pm 0,50)$ \\
\hline Bulimia e preocupação com alimentos & $2,25( \pm 0,19)$ & $1,88( \pm 0,22)$ \\
\hline Autocontrole oral & $4,75( \pm 0,27)^{\mathrm{b}}$ & $3,59( \pm 0,30)$ \\
\hline EAT-26 & $15,21( \pm 0,75)^{\mathrm{c}}$ & $11,32( \pm 0,83)$ \\
\hline
\end{tabular}

$\mathrm{M}=$ média; $\mathrm{DP}=$ desvio padrão.

${ }^{a} \mathrm{p}<0,05$ Diferença estatística na subescala dieta em relação ao sexo masculino; ${ }^{b} \mathrm{p}<0,05$ diferença estatística na subescala bulimia e preocupação com alimentos em relação ao sexo masculino; ${ }^{\mathrm{c}} \mathrm{p}<0,05$ diferença estatística na pontuação total do EAT-26 em relação ao sexo masculino.

Fonte: elaborada pelos autores. 
Remetendo-se as comparações do escore total e dos três fatores do EAT26 segundo os grupos de estado nutricional, foram encontradas diferenças significativas nas subescalas dieta $(F(4,358)=36,23, p=0,001)$ e bulimia $e$ preocupação com os alimentos $(F(4,358)=21,69, p=0,01)$, fato não reproduzido para a subescala autocontrole oral $(F(6,356)=2,99, p=0,16$, tabela 2$)$. Dessa forma, os adolescentes com sobrepeso e obesidade demonstraram maior frequência de restrição alimentar, comportamentos purgativos e compulsão alimentar em comparação aos com baixo peso e peso normal, embora a pressão percebida para a ingestão alimentar (subescala autocontrole oral) tenha sido semelhante entre os grupos de estado nutricional. Por fim, evidenciaram-se maiores pontuaçóes do EAT-26 em sujeitos com sobrepeso e obesidade $(F(4,358)=48,37, p=0,001$, tabela 2).

Tabela 2. Comparação do escore total e dos três fatores do EAT-26 de adolescentes de Juiz de Fora segundo estado nutricional

\begin{tabular}{|l|c|c|c|c|}
\hline \multicolumn{1}{|c|}{ Variável } & \multicolumn{4}{|c|}{ Estado nutricional } \\
\hline & $\begin{array}{c}\text { Baixo peso } \\
(n=17)\end{array}$ & $\begin{array}{c}\text { Peso normal } \\
(n=291)\end{array}$ & $\begin{array}{c}\text { Sobrepeso } \\
(n=37)\end{array}$ & $\begin{array}{c}\text { Obeso } \\
(n=17)\end{array}$ \\
\hline & $M(D P)$ & $M(D P)$ & $M(D P)$ & $M(D P)$ \\
\hline Dieta & $2,45( \pm 1,61)^{c, d}$ & $6,59( \pm 0,39)^{c, d}$ & $12,69( \pm 1,09)^{a, b}$ & $14,14( \pm 1,61)^{a, b}$ \\
\hline Bulimia e PA & $1,38( \pm 0,67)^{d}$ & $1,88( \pm 0,16)^{d}$ & $3,02( \pm 0,45)$ & $3,97( \pm 0,67)^{a, b}$ \\
\hline Autocontrole O & $5,08( \pm 0,92)$ & $4,08( \pm 0,22)$ & $4,20( \pm 0,62)$ & $5,92( \pm 0,92)$ \\
\hline EAT-26 & $9,12( \pm 2,62)^{c, d}$ & $12,26( \pm 0,63)^{c, d}$ & $20,00( \pm 1,77)^{a, b}$ & $24,06( \pm 2,62)^{a, b}$ \\
\hline
\end{tabular}

$\mathrm{M}=$ média; $\mathrm{DP}=$ desvio padrão; $\mathrm{PA}=$ preocupação com alimentos; $\mathrm{O}=$ oral.

${ }^{a} p<0,05$ em relação a baixo peso; ${ }^{b} p<0,05$ em relação a peso normal; ${ }^{c} p<0,05$ em relação a sobrepeso; ${ }^{d} p<0,05$ em relação a obeso.

Fonte: elaborada pelos autores.

\section{Discussão}

O estudo teve como premissa comparar fatores do comportamento alimentar entre os sexos e estado nutricional em adolescentes de Juiz de Fora-MG. Os resultados evidenciaram que o sexo feminino apresentou maior pontuação nas subescalas dieta e autocontrole oral, além de maior escore no EAT-26 quando comparado aos meninos. Ademais, adolescentes classificados com sobrepeso/ obesidade apresentaram pontuações nas subescalas dieta, bulimia e preocupação com os alimentos, e no EAT-26 maiores quando comparados aos jovens com baixo peso/peso normal.

Atualmente, são evidenciados resultados que demonstram aumento de prevalências de hábitos alimentares deletérios à saúde em jovens brasileiros 
(Castro et al., 2010; Dunker, Fernandes \& Carreira-Filho, 2009; Fortes et al., 2013; Gomes, Legnani, Legnani, Gregorio \& Souza, 2010; Martins et al., 2010; Oliveira \& Hutz, 2010; Rossi, Moreira \& Rauen, 2008; Scherer et al., 2010). Parece que os comportamentos alimentares inadequados têm se tornado hábito cotidiano na vida de adolescentes (Oliveira \& Hutz, 2010). No entanto o fato de o indivíduo apresentar alguns comportamentos alimentares inapropriados não remete necessariamente a síndromes psicológicas como os transtornos alimentares, mas torna esse sujeito mais vulnerável para o desenvolvimento de tais doenças (Martins et al., 2010; Rossi et al., 2008). Sabe-se que o sexo feminino e os jovens com estado nutricional inadequado (sobrepeso/obesidade) costumam ser mais acometidos por esses comportamentos (Alvarenga et al., 2011; Scherer et al., 2010). No entanto, até o momento, não se identificou estudo comparando construtos do comportamento segundo o sexo e o estado nutricional. Portanto, esta pesquisa destaca-se como inovadora ao propor esse tipo de análise com aspectos do comportamento alimentar no Brasil.

Os resultados deste estudo em relação à comparação das subescalas do EAT-26 segundo o sexo demonstraram que adolescentes do sexo feminino apresentaram maior frequência de recusa patológica alimentar e preocupação com a aparência física (dieta), além de maior magnitude de sentimentos de pressôes ambientais e sociais para a ingestão alimentar (autocontrole oral) quando comparadas aos seus pares do sexo masculino. Parece que jovens do sexo feminino se restringem com maior frequência a alimentos com alto teor calórico (Gomes et al., 2010). Talvez esse comportamento esteja associado à maior depreciação com o peso e a aparência física, geralmente encontrada nesse grupo (Duca et al. 2010; Miranda et al., 2011). Ademais, adolescentes femininas parecem estar mais susceptíveis para influências advindas de pais e amigos em comparação aos meninos (Alvarenga et al., 2011; Bernardi et al., 2009). Parece que adolescentes femininas costumam apresentar mais adaptações psicológicas negativas relacionadas aos comentários depreciativos sobre o peso e a aparência corporal, normalmente advindos de familiares e grupos sociais nos quais essas jovens estão inseridas (Rossi et al., 2008). Talvez por isso, adolescentes femininas desenvolvam comportamentos alimentares deletérios à saúde com o intuito de remodelamento corporal, na tentativa de serem aceitas socialmente (Alvarenga et al., 2011; Martins et al., 2010; Scherer et al., 2010). Em contrapartida, jovens do sexo masculino parecem não se preocupar tanto com os comentários negativos sobre seus corpos (Rossi et al., 2008), o que explica a diferença estatística encontrada na subescala autocontrole oral do EAT-26 entre os sexos.

Acreditava-se, todavia, que os sintomas de bulimia fossem diferentes entre os sexos. No entanto, nos achados deste estudo, não se identificou diferença 
dessa subescala do EAT-26 entre meninas e meninos, apontando atitudes semelhantes. Segundo Gomes, Legnani, Legnani, Gregorio e Souza (2010), a autoindução de vômitos e o uso de medicamentos (laxantes e diuréticos) visando ao emagrecimento não são comportamentos prioritários do sexo feminino, podendo serem identificados na mesma proporção entre os meninos. Entretanto outros pesquisadores salientam que o sexo feminino é mais acometido por hábitos purgativos quando comparado a grupos masculinos (Bernardi et al., 2009; Martins et al., 2010). Talvez essas discrepâncias de resultados e hipóteses estejam associadas à variedade de instrumentos utilizados em pesquisas e, ou, a variabilidade cultural presente entre as cidades e os Estados brasileiros, devendose atentar para essas questôes quando se compararam evidências de pesquisas científicas. Ressalta-se, portanto, a necessidade de mais investigaçōes acerca desses comportamentos entre os jovens do sexo masculino, a fim de identificar os fatores mais fortemente relacionados à etiologia dos comportamentos alimentares inadequados nesse público.

Remetendo-se à comparação das subescalas do EAT-26 em razão do estado nutricional, os resultados desta pesquisa evidenciaram que adolescentes com sobrepeso/obesidade apresentaram maior frequência de recusa patológica alimentar e preocupação com a forma física quando comparados a jovens com baixo peso/peso normal. Parece que a cultura do corpo magro, disseminada pela mídia, afeta mais os adolescentes que apresentam excesso de peso (Alvarenga et al., 2011; Gomes et al., 2010; Petroski, Pelegrini \& Glaner, 2009; Scherer et al., 2010). Talvez por isso, jovens com sobrepeso/obesidade preocupem-se mais com o peso e a aparência física. No entanto a recusa patológica alimentar mais frequente em relação aos adolescentes com baixo peso/peso normal não se justifica. Provavelmente porque a subescala dieta do EAT-26 não separa a avaliação dos dois quesitos (recusa patológica alimentar e preocupação com forma física), torna-se inadequado falar que adolescentes com sobrepeso/obesidade apresentem recusa patológica alimentar mais acentuada quando comparados a jovens eutróficos. No entanto, sujeitos classificados com obesidade demonstraram maior frequência de comportamentos bulímicos quando comparados a indivíduos com baixo peso/ peso normal. Esses achados estão de acordo com o que a literatura preconiza, ou seja, jovens com sintomas de bulimia costumam apresentar excesso de peso (Alvarenga et al., 2011; Duca et al., 2010; Martins et al., 2010; Rossi et al., 2008). Em contrapartida, não foi encontrada diferença na subescala autocontrole oral entre os grupos de estado nutricional, contrapondo as hipóteses apresentadas por outros autores de que os jovens com sobrepeso/obesidade costumam sofrer maiores pressões de familiares e amigos a respeito da atenuação de peso corporal (Bernardi et al., 2009; Gomes et al., 2010; Pelegrini \& Petroski, 2009). Talvez 
as influências negativas parentais e de grupos sociais sejam semelhantes entre os jovens com diferentes estados nutricionais na cidade de Juiz de Fora-MG.

Este estudo demonstrou resultados inéditos e interessantes para o campo científico. No entanto, esta pesquisa apresentou limitações que merecem ser apontadas. Em primeiro lugar, o uso de uma escala autoaplicável para avaliar comportamentos alimentares. Alguns autores ressaltam que ferramentas autorreportadas podem mascarar a realidade do fenômeno no contexto avaliado, pois se tratam de respostas subjetivas. No entanto, Miranda Conti, Bastos e Ferreira (2011) salientam que, em estudos epidemiológicos ou de grandes amostras, os questionários são instrumentos que podem ser considerados "padrão ouro", pois não necessitam de mão de obra especializada para sua aplicação, além de requisitar baixo custo operacional. A segunda limitação foi utilizar o IMC como ponto de corte para classificar o estado nutricional. Pesquisadores afirmam que o IMC pode classificar erroneamente sujeitos com massa muscular acentuada como obesos (Pelegrini \& Petroski, 2009). Por outro lado, Castro, Levy, Cardoso, Passos, e Sardinha (2010) ressaltam que o IMC deve ser utilizado em pesquisas com tamanho amostral elevado, como é o caso deste estudo. Por fim, este estudo evidenciou achados que merecem ser discutidos na literatura científica, pois se acredita que contemple análises que até então não haviam sido exploradas.

\section{Conclusões}

Os resultados da pesquisa permitem concluir que a recusa patológica alimentar e as pressóes ambientais e sociais foram diferentes entre os sexos. Ademais, jovens com sobrepeso/obesidade apresentaram preocupação com a forma física e sintomas de bulimia mais frequentes quando comparados ao grupo com baixo peso/peso normal. Todavia vale destacar que $82 \%$ dos adolescentes do sexo masculino e $70 \%$ do feminino não demonstraram comportamentos alimentares inadequados.

São sugeridos outros estudos com jovens brasileiros na tentativa de identificar possíveis fatores de risco para os comportamentos alimentares inadequados, investigando os demais fatores envolvidos na manifestação desse fenômeno. 


\section{Referências}

Alvarenga, M. S., Scagliusi, F. B. \& Phillippi, S. T. (2011). Comportamento de risco para transtorno alimentar em universitárias brasileiras. Revista de Psiquiatria Clinica, 38(1), 3-7.

Bernardi, F., Harb, A. N. C., Levandovski, R. M. \& Hidalgo, M. P. L. (2009). Transtornos alimentares e padrão circadiano alimentar: uma revisão. Revista de Psiquiatria do Rio Grande do Sul, 31(3), 170-176.

Bighetti, F., Santos, C. B., Santos, J. E. \& Ribeiro, R. P. P. (2004). Tradução e avaliação do Eating Attitudes Test em adolescentes do sexo feminino de Ribeirão Preto, São Paulo. Jornal Brasileiro de Psiquiatria, 53(6), 339-346.

Castro, I. R. R., Levy, R. B., Cardoso, L. O., Passos, M. D. \& Sardinha, L. M. V. (2010). Imagem corporal, estado nutricional e comportamento com relação ao peso entre adolescentes brasileiros. Ciência \& Saúde Coletiva, 15(supl. 2), 3099-3108.

Duca, G. F. D., Garcia, L. M. T., Sousa, T. F., Oliveira, E. S. A. \& Nahas, M. V. (2010). Insatisfação com o peso corporal e fatores associados em adolescentes. Revista Paulista de Pediatria, 28(4), 340-346.

Dunker, K. L. L., Fernandes, C. P. B. \& Carreira-Filho, D. (2009). Influência do nível socioeconômico sobre comportamentos de risco para transtornos alimentares em adolescentes. Jornal Brasileiro de Psiquiatria, 58(3), 156-161.

Ekeroth, K. \& Birgegård, A. (2014). Evaluating reliable and clinically significant change in eating disorders: Comparisons to changes in DSM-IV diagnoses. Psychiatry Research, 216(2), 248-254

Fortes, L. S., Almeida, S. S., Cipriani, F. M. \& Ferreira, M. E. C. (2014). Comportamento alimentar inadequado: uma investigação longitudinal com adolescentes do sexo feminino. Revista Paulista de Pediatria, 32(1), 85-91.

Fortes, L. S., Amaral, A. C. S., Conti, M. A., Cordás, T. A. \& Ferreira, M. E. C. (2016) Qualidades psicométricas do Eating Attitudes Test (EAT-26) para a população adolescente brasileira masculina. Psicologia: Reflexão e Crítica, no prelo.

Fortes, L. S., Kakeshita, I. S., Gomes, A. R., Almeida, S. S. \& Ferreira, M. E. C. (2014). Eating behaviours in youths: a comparison between female and male athletes and non-athletes. Scandinavian Journal of Medicine \& Science in Sports, 24(1), e62-e68. 
Fortes, L. S., Morgado, F. F. R. \& Ferreira, M. E. C. (2013). Fatores associados ao comportamento alimentar inadequado em adolescentes escolares. Revista de Psiquiatria Clínica, 40(2), 59-64.

Garner, D. M., Olmsted, M. P., Bohr, Y. \& Garfinkel, P. A. (1982). The eating attitudes test: psychometric features and clinical correlations. Psychology Medicine, 12(1), 871-878.

Gomes, J. P., Legnani, E., Legnani, R. F. S., Gregorio, N. P. \& Souza, R. K. (2010). Associação entre comportamento alimentar, consumo de cigarro, drogas e episódios depressivos em adolescentes. Revista de Nutrição, 23(5), 755-762.

Johnson, C., Crosby, R., Engel, S., Mitchell, J. \& Powers, P. (2004). Gender, ethnicity, self-esteem and disordered eating among college athletes. Eating Behaviors, 5(1), 147-156.

Martins, C. R., Pelegrini, A., Matheus, S. C. \& Petroski, E. L. (2010). Insatisfação com a imagem corporal e a relação com estado nutricional, adiposidade corporal e sintomas de anorexia e bulimia em adolescentes. Revista de Psiquiatria do Rio Grande do Sul, 32(1), 19-23.

Minas Gerais. Secretaria de Educação de Minas Gerais (2011). Senso escolar do ano de 2010. Belo Horizonte: Secretaria de Educação do Estado de Minas Gerais. Recuperado a partir de: https://www.educacao.mg.gov.br.

Miranda, V. P. N., Conti, M. A., Bastos, R. \& Ferreira, M. E. C. (2011). Insatisfação corporal em adolescentes brasileiros de municípios de pequeno porte de Minas Gerais. Jornal Brasileiro de Psiquiatria, 60(3), 190-197.

Oliveira, L. L. \& Hutz, C. S. (2010). Transtornos alimentares: o papel dos aspectos culturais no mundo contemporâneo. Psicologia em Estudo, 15(3), $575-582$.

Pelegrini, A. \& Petroski, E. L. (2009). Inatividade física e sua associação com estado nutricional, insatisfação com a imagem corporal e comportamentos sedentários em adolescentes de escolas públicas. Revista Paulista de Pediatria, 27(4), 366-373.

Petroski, E. L., Pelegrini, A. \& Glaner, M. F. (2009). Insatisfação corporal em adolescentes rurais e urbanos. Motricidade, 5(4),13-25. 
Rossi, A., Moreira, E. A. D. \& Rauen, M. S. (2008). Determinantes do comportamento alimentar: uma revisão com enfoque na família. Revista de Nutrição, 21(6), 739-748.

Santos, E. M. C., Tassitano, R. M., Nascimento, W. M. F., Petribu, M. M. V. \& Cabral, P C. (2011). Satisfação com o peso corporal e fatores associados em adolescentes do ensino médio. Revista Paulista de Pediatria, 29(2), 214-223.

Scherer, F. C., Martins, C. R., Pelegrini, A., Matheus, S. C. \& Petroski, E. L. (2010). Imagem corporal em adolescentes: associação com a maturação sexual e sintomas de transtornos alimentares. Jornal Brasileiro de Psiquiatria, 59(3), 198-202.

Siervogel, R. M., Dmerath, E. W., Schubert, C., Remsberg, K. E. \& Chumlea, W. C. (2003). Puberty and body composition. Hormone Research, 60(1), 3645 .

Ximenez, R. C. C., Colares, V., Bertulino, T., Couto, G. B. L. \& Sougey, E. B. (2011). Versão brasileira do BITE para uso em adolescentes. Arquivos Brasileiros de Psicologia, 63(1), 52-63.

World Health Organization (2007). Development of a WHO growth reference for school-aged children and adolescents. Bulletin of the World Health Organization, 85(9), 660-667. 